\title{
Controlling Lease Time in Dynamic Host Configuration Protocol Servers
}

\author{
Tien Van Do \\ Department of Telecommunications, \\ Budapest University of Technology and Economics \\ H-1117, Magyar tudósok körútja 2., Budapest, Hungary, \\ Email: do@hit.bme.hu
}

\begin{abstract}
Dynamic Host Configuration Protocol (DHCP) allows the automatic networking configuration of computers and devices (clients) in Internet Protocol (IP) networks. It is used by clients to request an IP address and obtain configuration parameters (netmask, router IP address, Domain Name Server -DNS- address etc.) for IP networking from a DHCP server. For this purpose, a pool of IP addresses is administered and maintained in a DHCP server. In order to reuse an IP address that is no longer needed by the client to which it was assigned, a lease time parameter is applied. That is, each client leases an IP address from the chosen DHCP server for a limited period of time.
\end{abstract}

In this paper, we present a performability model for the allocation of IP addresses in DHCP servers. We also illustrate the impact of the lease time parameter on the allocation of IP addresses and the number of clients waiting for the allocation of an IP address.

Keywords: DHCP, IP address allocation, lease time, performability

\section{INTRODUCTION}

IP address is a scarce resource in Internet and an Internet Service Provider environment. DHCP is one of many techniques to handle this issue because it supports the reassignment of IP network addresses. In addition, it provides an efficient method to automate and centrally manage the network of computers and network devices [3].

Dynamic Host Configuration Protocol (DHCP) is designed by the dynamic host configuration working group within the framework of the Internet Engineering Task Force (IETF). At the present, DHCP is specified for Internet Protocol version 4 in IETF "draft standard" RFC 2131 [2] and for Internet Protocol version 6 in IETF RFC 4361 [6]. The main aim of DHCP is to provide an automatic mechanism for the allocation, configuration and management of IP addresses and TCP/IP protocol stack parameters for the participating computers and devices in IP networks. That is, DHCP is used by clients to obtain configuration parameters for IP networking (IP address, netmask, router IP address, etc.) from DHCP servers.

The important feature of DHCP is a "dynamic allocation" mechanism, which assigns an IP address to a client for a limited period of time (called a lease time). Therefore, a previously allocated IP address which is not used by one host can automatically be assigned to another host by a DHCP server implementing the dynamic allocation mechanism. It is recognized that the appropriate setting of a lease time in a DHCP server plays an important role in the efficient allocation of IP addresses. In [4], the authors investigated the impact of setting lease times using the data from the Georgia Tech campus network. However, due to the lack of a quantitative performability model and the lack of data at clients (whether they are forced to wait for an IP address), they only could examine the utilization of the allocatable address space in a DHCP server.

This paper proposes a method to quantitatively evaluate the performance of a DHCP dynamic allocation mechanism and the impact of a lease time. To construct a retrial queue and a tractable solution, the following steps are performed. We show that interarrival times of DHCP requests from clients follow the exponential distribution. We make a relaxation assumption concerning the lease time sent by a DHCP server and the retrials of clients. It is shown via simulation of more detailed model than an analytical abstract model of DHCP that the proposed model is accurate to calculate the performance of the interaction 
between the behavior of clients and the DHCP mechanism. A numerical study is also performed, which provides an insight for the impact of trade-off parameters and factors on the operation of DHCP.

The rest of this paper is organized as follows. In Section II, the overview of DHCP operation is presented. In Section III, the proposed model is described. In Section IV a numerical study is provided to validate the computational approach and reveal some interesting behaviors of the IP address allocation mechanism. Finally, the paper is concluded in Section V.

\section{DHCP OPERATION}

DHCP follows a client-server model. A DHCP server centrally manages a range of IP addresses and parameters specified by network administrators for a specific IP subnet, which can be allocated to hosts. A DCHP client software running on computers or devices requests information from a DHCP server. The communications between a DHCP server and a client are delivered by the DHCP protocol.

The important types of the DHCP protocol messages include DHCPDISCOVERY, DHCPOFFER and DHCPREQUEST. DHCPDISCOVERY messages are sent in a broadcast UDP packet by client hosts to find available servers. A DHCP server may check (e.g.: based on the MAC address of a client) whether a client is authorized to request an IP address upon the arrival of a DHCPDISCOVER messsage, which is dependent on the configuration of a DHCP server. If a client is authorized to request an IP address or if no authorization is performed, the DHCP server sends DHCPOFFER message with configuration parameters (IP address, netmask, router address, DNS server address). Note that the whole process is performed in the similar way, if a client knows the IP address of a DHCP server in advance of the request of an IP address. The only exception is that a client sends DHCPREQUEST message instead of DHCPDISCOVERY message.

Three main modes for IP address allocation are supported: manual, automatic and dynamic allocation. The purpose of the "manual allocation" mode is to allow the network administrator to centrally store information concerning client hosts. In this mode the IP address is assigned by the network operator to a client host. After the identification of a specific client (e.g., based on hardware MAC address) DHCP sends a fixed IP address and configuration parameters (e.g.: the subnet mask, the default gateway address) for the client. This kind of operation is typically applied in a campus or LAN environment. In the mode "automatic allocation", a DHCP server assigns a permanent IP address to a client hosts.

The most important feature of DHCP is the "dynamic allocation", where an IP address is assigned to a client for a limited period of time. A lease time is defined as a period of time for which the server gives a permission for a client to use the address. Note that a lease time is also sent to a client. Upon the expiration of the lease time, the allocated address becomes free and can be assigned to another client unless a client extends the use of a specific IP address before the expiration of the lease time. This feature is often applied in the environment of Internet Service Providers because the reuse and sharing of scarce IP addresses is possible.

The decision that a DHCP client "leaves" the system or renews the use of the allocate IP address depends on the relation between the lease time and the holding time (e.g.: the working time) of clients. In order to extend the use of the allocated IP address the client sends a DHCPREQUEST message which includes the client's allocated IP address in the "requested IP address" option of a DHCPREQUEST message.

\section{QUeUeING MODEL FOR THE ALLOCATION OF IP ADDRESSES}

We draw a queueing model for the allocation of IP addresses in a DHCP server in Fig. 1, in which IP addresses are modeled as service facilities (i.e. servers). In the queueing model, an arriving DHCP request is allocated an IP address if there is a free IP address in the pool. A client who does not receive the allocation of an IP address because the shortage of IP addresses sets a timer to wait for a limited time and will retry the request for an IP address upon the expiration of backoff time. We model this phenomenon as a client joins the "virtual orbit" and waits in the orbit to retry the request. 


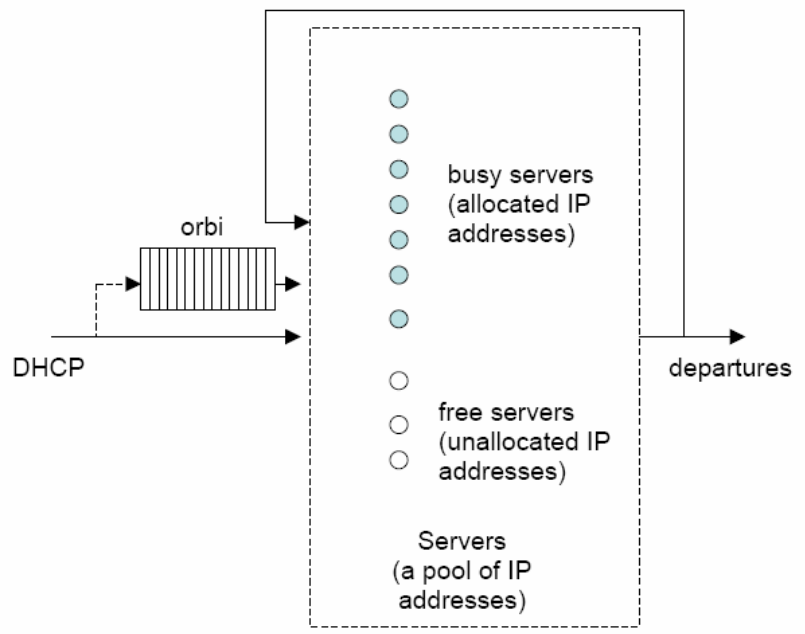

Fig. 1. Queueing model for the allocation of IP addresses

\section{III.1. A Retrial Queue}

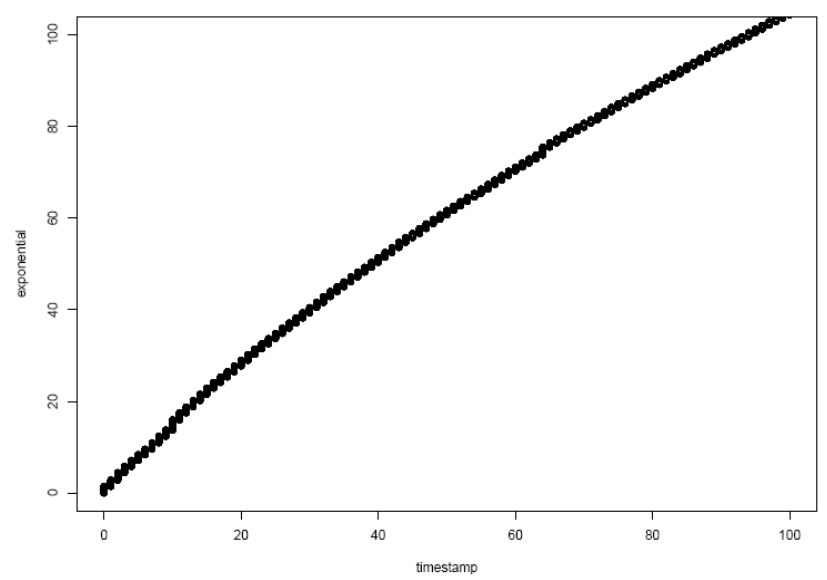

Fig. 2. Q-Q plot for the interarrival times (measured in seconds) of DHCPDISCOVERY messages

We assume the interarrival times of DHCP DISCOVERY messages are exponentially ${ }^{1}$ distributed with a parameter $\lambda$.

The size of the pool (i.e.: the number of allocatable IP addresses) is $c$. Let $I(t)$ denote the number of

We process the log file of the DHCP server of our department between the period of January 2 and May 28, 2008. The DHCP server serves 80 people, half of them use laptops requesting IP addresses from the DHCP server. In Fig. 2, the straight line of the Q-Q plot, where the interarrival times of DHCP requests between $8 \mathrm{~h}$ and $18 \mathrm{~h}$ during the investigation period to the DHCP server are plotted against the theoretical exponential distribution, confirms our assumption. allocated IP addresses at time $t$. The minimum value of $I(t)$ is 0 and $c$, the maximum.

There are two possibilities concerning a client that successfully receives an IP address. Upon the expiration of the lease time (denoted by $T_{l}$ ), the previously allocated address at the DHCP server becomes free and can be allocated to another client unless the client extends the use of a specific IP address before the expiration of the lease time. Assume that DHCP clients leave (i.e.: switch off the computer) the system or do not renew the allocated IP address with probability $a$ $(a>0)$ after the expiration of its lease time.

A client who does not receive the allocation of an IP address because the shortage (when $I(t)=c$ ) of IP addresses sets a timer to wait for a limited time and will retry the request for an IP address upon the expiration of backoff time. We model this phenomenon as the client joins the "virtual orbit". $J(t)$ represents the number of DHCP clients in the "orbit" at time $t$ and takes values from 0 to $\infty$.

In order to have a tractable queueing system, we assume that lease times are exponentially distributed with parameter $\mu$ and clients waiting in the orbit repeat the request for the DHCP server with rate $v$ (i.e.: the inter-repetition times are exponentially distributed with parameter $v$ ). As a consequence, the system is modeled by a CTMC, $Y=\{I(t), J(t)\}$, with a state space $\{0,1, \ldots, c\} \times\{0,1, \ldots\}$.

\section{2. A Quasi-Birth-and-Death (QBD) representation}

We denote the steady state probabilities by $\pi_{i, j}=\lim _{t \rightarrow \infty} \operatorname{Prob}(I(t)=i, J(t)=j) \quad$, and introduce $\mathbf{v}_{j}=\left(\pi_{0, j}, \ldots, \pi_{c, j}\right)$.

The evolution of $Y$ is driven by the following transitions.

(a) $A_{j}(i, k)$ denotes a transition rate from state $(i, j)$ to state $(k, j)(0 \leq i, k \leq c ; j=0,1, \ldots)$. 


$$
A_{j}=A=\left[\begin{array}{ccccccc}
0 & \lambda & 0 & \ldots & 0 & 0 & 0 \\
a \mu & 0 & \lambda & \ldots & 0 & 0 & 0 \\
\vdots & \vdots & \vdots & \vdots & \vdots & \vdots & \vdots \\
0 & 0 & & \ldots & a(c-1) \mu & 0 & \lambda \\
0 & 0 & & \ldots & 0 & a c \mu & 0
\end{array}\right] \forall j \geq 0
$$

(b) $B_{j}(i, k)$ represents one step upward transition from state $(i, j)$ to state $(k, j+1)$ $(0 \leq i, k \leq c ; j=0,1, \ldots)$.

$$
B_{j}=B=\left[\begin{array}{ccccccc}
0 & 0 & 0 & \ldots & 0 & 0 & 0 \\
0 & 0 & 0 & \ldots & 0 & 0 & 0 \\
\vdots & \vdots & \vdots & \vdots & \vdots & \vdots & \vdots \\
0 & 0 & & \ldots & 0 & 0 & 0 \\
0 & 0 & & \ldots & 0 & 0 & \lambda
\end{array}\right] \forall j \geq 0
$$

(c) $C_{j}(i, k)$ is the transition rate from state $(i, j)$ to state $(k, j-1)(0 \leq i, k \leq c ; j=0,1, \ldots)$.

$$
C_{j}=C=\left[\begin{array}{ccccccc}
0 & v & 0 & \ldots & 0 & 0 & 0 \\
0 & 0 & v & \ldots & 0 & 0 & 0 \\
\vdots & \vdots & \vdots & \vdots & \vdots & \vdots & \vdots \\
0 & 0 & & \ldots & 0 & 0 & v \\
0 & 0 & & \ldots & 0 & 0 & 0
\end{array}\right] \forall j \geq 1
$$

We introduce diagonal matrices $D^{A}$ and $D^{C}$. The diagonal elements are the sum of the elements in the row of $A$ and $C$. The infinitesimal generator matrix of $Y$ can be written as follows

$$
\left[\begin{array}{ccccccc}
A_{00} & B & 0 & \ldots & \ldots & \ldots & \ldots \\
C & Q_{1} & B & 0 & \ldots & \ldots & \ldots \\
0 & C & Q_{1} & B & 0 & \ldots & \ldots \\
0 & 0 & C & Q_{1} & B & 0 & \ldots
\end{array}\right]
$$

where $A_{00}=A-D^{A}-B$ and $Q_{1}=A-D^{A}-B-D^{C}$.

Because of the structure of the QBD, the steady state probabilities can be obtained with the existing methods like the matrix-geometric and its variants $[1,5,8]$, and the spectral expansion [7].
IV. EVALUATION OF THE DHCP MECHANISM IV.1. Evaluation of the DHCP mechanism

In order to have a mathematically tractable model, we have assumed that the lease time values sent by a DHCP server follow the exponential distribution. However, a lease time sent by a specific DHCP server to clients is of fix value.

Assume that the holding times (i.e.: how long does a client need an IP address) of clients have a distribution function $F(x)$ and the fix lease time value sent by a DHCP is $T_{l}$. Then, we apply the retrial queue in Section III.1 with $\mu=1 / T_{l}$ and $a=F\left(T_{l}\right)$ to evaluate the performance of the DHCP dynamic allocation mechanism. It will be shown through the comparison with the simulation of the real DHCP allocation mechanism that this assumption has almost no impact on the evaluation of the performance measures of the DHCP dynamic allocation mechanism.

Note that performance parameters related to the DHCP dynamic allocation mechanism are obtained as follows:

average number of occupied IP addresses

$$
N_{\text {occ }}=\sum_{i=1}^{c} i \sum_{j=0}^{\infty} \pi_{i, j}
$$

average number of clients waiting in the orbit

$$
N_{\text {orbit }}=\sum_{j=1}^{\infty} j \sum_{i=0}^{c} \pi_{i, j}
$$

We also present the validation of our model with simulation. It is worth emphasizing that the simulation model captures the two aspects of a real DHCP server in a better way than the analytical one. The simulation model is constructed to be close to the operation of the DHCP mechanism and the behavior of users. That is, the lease time sent to each a client is of a fixed value in a specific simulation and each client independently retries an IP requests after 30 seconds (it is the normal value observed in a DHCP client software implemented in the present operating systems). The simulation model is implemented in a program using $C$ language.

The simulation results are generated with the confident level of 99\%. As observed from Table 1 the 
agreement between the simulation and analytical results is excellent.

\begin{tabular}{|c|c|c|c|c|}
\hline \multicolumn{5}{|c|}{ Lease time: 5 minutes } \\
\hline Average & \multicolumn{2}{|c|}{ Analytical Model } & \multicolumn{2}{|c|}{ Simulation (conf. level=99\%) } \\
\hline $\begin{array}{l}\text { time } \\
\text { (minutes) }\end{array}$ & осс $N$ & orbit $N$ & oсc $N$ & orbit $N$ \\
\hline 10 & 12.7075 & 0 & 12.715630 & 0 \\
\hline 30 & 32.5694 & 0 & 32.590180 & 0 \\
\hline 60 & 62.5347 & 0 & 62.574540 & 0 \\
\hline 90 & 92.5231 & 0 & 92.582617 & 0 \\
\hline 120 & 122.5170 & 0 & 122.596091 & 0 \\
\hline 150 & 152.5140 & 0 & 152.612031 & 0 \\
\hline 180 & 182.5120 & 0.000004 & 182.628786 & 0.000002 \\
\hline & & \multicolumn{2}{|c|}{ Lease time: 30 minutes } & \\
\hline 10 & 31.5719 & \multicolumn{2}{|c|}{031.591576} & 0 \\
\hline 30 & 47.4593 & \multicolumn{2}{|c|}{047.490397} & 0 \\
\hline 60 & 76.2448 & \multicolumn{2}{|c|}{076.293760} & 0 \\
\hline 90 & 105.832 & \multicolumn{2}{|c|}{0105.899821} & 0 \\
\hline 120 & 135.624 & \multicolumn{2}{|c|}{0135.709828} & 0 \\
\hline 150 & 165.500 & \multicolumn{2}{|c|}{0165.605376} & 0 \\
\hline 180 & 195.416 & \multicolumn{2}{|c|}{0.000437195 .540905} & 0.000423 \\
\hline & & \multicolumn{2}{|c|}{ Lease time: 60 minutes } & \\
\hline 10 & 60.1491 & \multicolumn{2}{|c|}{060.186734} & 0 \\
\hline 30 & 69.3911 & \multicolumn{2}{|c|}{069.436389} & 0 \\
\hline 60 & 94.9186 & \multicolumn{2}{|c|}{094.980781} & 0 \\
\hline 90 & 123.309 & \multicolumn{2}{|c|}{0123.385416} & 0 \\
\hline 120 & 152.49 & \multicolumn{2}{|c|}{0152.587474} & 0 \\
\hline 150 & 181.995 & \multicolumn{2}{|c|}{0182.111794} & \\
\hline 180 & 211.664 & \multicolumn{2}{|c|}{0.03816211 .799544} & 0.033517 \\
\hline & & \multicolumn{2}{|c|}{ Lease time: 90 minutes } & \\
\hline 10 & 90.0111 & 0 & 90.068141 & 0 \\
\hline 30 & 94.7156 & 0 & 94.774729 & 0 \\
\hline 60 & 115.850 & 0 & 115.921970 & 0 \\
\hline 90 & 142.378 & 0 & 142.471148 & 0 \\
\hline 120 & 170.573 & 0 & 170.679458 & 0 \\
\hline 150 & 199.473 & 0.001519 & 199.600627 & 0.001356 \\
\hline \multirow[t]{2}{*}{180} & 228.734 & 1.299020 & 228.881151 & 1.016362 \\
\hline & & \multicolumn{2}{|c|}{ Lease time: 120 minutes } & \\
\hline 10 & 120.001 & \multicolumn{2}{|c|}{0120.076530} & 0 \\
\hline 30 & 122.239 & \multicolumn{2}{|c|}{0122.315397} & 0 \\
\hline 60 & 138.782 & \multicolumn{2}{|c|}{0138.872755} & 0 \\
\hline 90 & 162.954 & \multicolumn{2}{|c|}{0163.052606} & 0 \\
\hline 120 & 189.837 & 0.000 & 189.961514 & 0.000060 \\
\hline 150 & 217.916 & 0.154 & 218.052797 & 0.114559 \\
\hline 180 & 246.618 & 67.667 & 247.106000 & 66.585000 \\
\hline
\end{tabular}

Table 1. Analytical and simulation results ( $c=250, \lambda=1 / 60$ requests $/ \mathrm{s}$ )

\section{IV.2. Numerical results}

To illustrate the impact of a lease time on the performability of the DHCP server, we present some numerical results. In the case study, IP address assignments arrive with a rate of 5 requests/minute. The size of the pool of IP addresses available at the DHCP server is 1000 . We plot the average number of occupied IP addresses versus the average holding time and the lease time in Fig. 3, and the average number of requests waiting in the orbit versus the average holding time and the lease time in Fig. 4. It is seen that the system is overloaded when the average holding time is higher than 200 minutes.

The most important resource of the DHCP server is the pool of IP address, so the efficient allocation of IP address poses a crucial issue for the network administrator. As one observes that the allocation of IP addresses can be controlled with the appropriate setting of the lease length. If the DHCP is not overloaded, then the smaller the lease time is, the more efficient the allocation of IP address (Fig. 3) and the smaller the number of requests waiting in the orbit is (Fig. 4). For example when the average holding time is 90 minutes and a lease time has a value of 30 minutes, the average number of occupied IP addresses is 529 (471 free IP addresses are available in average). If we change the setting of a lease time to 120 minutes, only 186 free IP addresses are available in a DHCP server. It is worth emphasizing that the small value setting of the lease time has the impact of increased number (load) of renewal messages (DHCPREQUEST). Note that in this case, the rate of DHCPREQUEST renewal messages can be easily handled by a DHCP server running in commodity hardware, which is less than 5 renewals/minute.

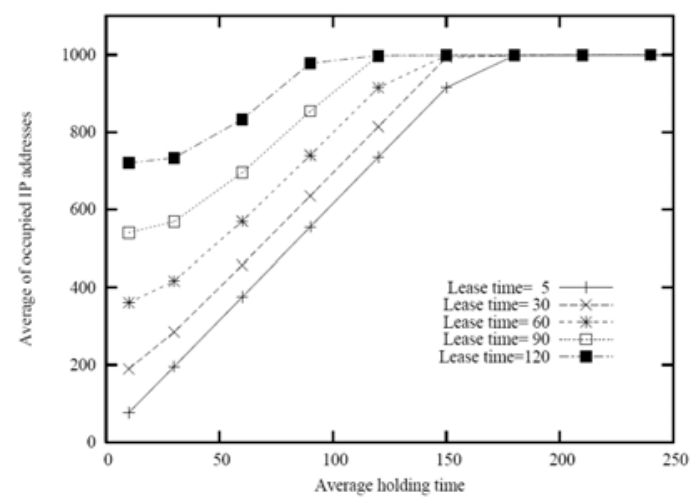

Fig. 3. Average number of occupied IP addresses

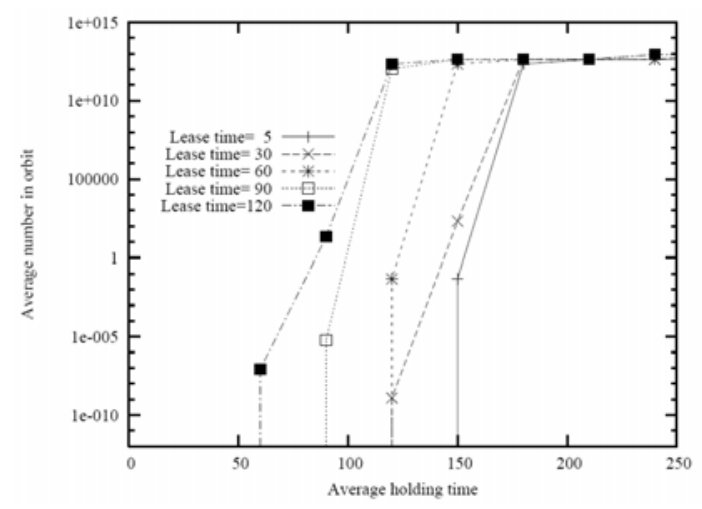

Fig. 4. Average number of requests waiting in the orbit 


\section{CONCLUSIONS}

We have presented the queueing model and computational approach to model the dynamic allocation mechanism of DHCP.

We have observed that the setting of a small lease time in a DHCP server has the advantage of the more efficient usage (i.e.: more clients can be allocated) of the IP address pool and the smaller number of clients waiting in the orbit than a large lease time. It is also worth emphasizing that we also have to take into account the load of renewal messages when we want to set a small lease time (i.e: a DHCP server is powerful enough to handle renewal messages). However, the contra argument against the small lease time is the policy enforced by the service. That is, they may not assign the same IP address when a DHCP client sends the renewal message, which will cause an interruption for some services (e.g.: the termination of an ongoing VoIP call).

Our model can be a useful tool for the optimal setting of the lease time given the average holding time, request arrival rate users, size of IP address pool, the processing capacity of the DHCP servers and the policy of the service provider.

\section{ACKNOWLEDGEMENT}

The author thanks the reviewers for the constructive comments.

\section{REFERENCES}

[1] D. Bini and B. Meini. On the solution of a nonlinear matrix equation arising in queueing problems. SIAM Journal on Matrix Analysis and Applications, 17(4):906926, 1996.

[2] R. Droms. Dynamic Host Configuration Protocol. RFC 2131 (Draft Standard), March 1997. Updated by RFCs 3396, 4361.

[3] R. Droms and T. Lemon. The DHCP Handbook, second edition. Sams, 2002.

[4] Manas Khadilkar, Nick Feamster, Matt Sanders, and Russ Clark. Usage-based dhcp lease time optimization. In IMC '07: Proceedings of the 7th ACM SIGCOMM conference on Internet measurement, pages 71-76, New York, NY, USA, 2007. ACM.

[5] Guy Latouch and V. Ramaswami. A logarithmic reduction algorithm for quasi-birth-death processes. Applied Probability, pages 650-674, 1993.

[6] T. Lemon and B. Sommerfeld. Node-specific Client Identifiers for Dynamic Host Configuration Protocol
Version Four (DHCPv4). RFC 4361 (Proposed Standard), February 2006.

[7] I. Mitrani and R. Chakka. Spectral expansion solution for a class of Markov models: Application and comparison with the matrix-geometric method. Performance Evaluation, 23:241-260, 1995.

[8] V. Naoumov, U. Krieger, and D. Wagner. Analysis of a Multi-server Delay-loss System with a General Markovian Arrival Process. In S.R. Chakravarthy and A.S. Alfa, editors, Matrix-analytical methods in Stochastic models, pages 43-66. Marcel Dekker, 1997.

\section{AUTHOR BIOGRAPHY}

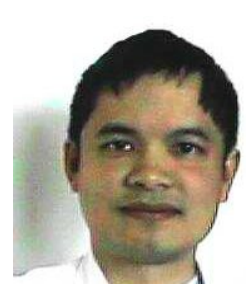

Tien Van Do received the M.Sc. and Ph.D. degrees in telecommunications engineering from the Technical University of Budapest, Hungary, in 1991 and 1996, respectively. He is an associate professor in the Department of Telecommunications of the Technical University of Budapest, and a leader of Communications Network Technology and Internetworking Group. He has participated in the COPERNICUS-ATMIN 1463, the FP4 ACTS AC310 ELISA, FP5 HELINET, FP6 CAPANINA projects funded by EC, and lead various projects on network planning, software implementations (ATM \& IP network planning software, GGSN tester, program for IMS performance testing, VoIP measurement,...), test and performance evaluation with NOKIA, T-COM, NOKIA and Siemens Networks, and industry partners. He was the person in charge for the RFI (Request for Information) and the technical specification of the public procurement worth of 2 MEuro for the testbed (IMS, UMTS, WiFi, etc,...) of Mobile Innovation Center in Budapest. His research interests are queuing theory, telecommunication networks, performance evaluation and planning of telecommunication networks. 